\title{
Desenvolvimento da trombose em pacientes com e sem infecção pelo SARS-Cov-2 -
}

\section{revisão de literatura}

\author{
Development of thrombosis in patients with and without SARS-Cov-2 infection - literature review \\ Desarrollo de la trombosis en pacientes con y sin infección por SARS-Cov-2 - revisión de la \\ bibliografía
}

Recebido: 08/11/2021 | Revisado: 16/11/2021 | Aceito: 17/11/2021 | Publicado: 24/11/2021

\author{
Luciana Da Silva Tavares \\ ORCID: https://orcid.org/0000-0003-1295-2511 \\ Universidade Nilton Lins, Brasil \\ E-mail: Luciana_lulis2010@hotmail.com \\ Jessica Vanina Ortiz \\ ORCID: https://orcid.org/0000-0001-7214-6816 \\ Universidade Nilton Lins, Brasil \\ E-mail: ortiz.jvm@gmail.com
}

\begin{abstract}
Resumo
A trombose venosa é caracterizada pelo acúmulo excessivo de estruturas compostas por fibrinas e plaquetas no interior de veias, ocasionando obstrução parcial ou oclusão, afetando geralmente os membros inferiores. Destacam-se como principais fatores de risco a estase sanguínea, lesão endotelial e estados de hipercoagulabilidade, intensificandose com o avançar da idade. Correlacionar o desenvolvimento da trombose em pacientes sadios e com os que foram acometidos com a COVID-19. A busca de artigos científicos foi realizada nas bases de dados eletrônicas: Scientific Electronic Library Online (SCIELO), Literatura Latino-Americana em Ciências da Saúde (LILACS), National Library of Medicine and the National Institute of Health (Pubmed). Em se tratando da ocorrência de eventos trombóticos na COVID-19, acredita-se que a trombose seja desencadeada pela "tempestade de citocinas" que é a presença de um nível exacerbado de citocinas pró-inflamatória no sangue. Pelo fato de muitas afecções apresentarem, ao exame clínico, quadro semelhante aos encontrados em pacientes de trombose, é necessária a realização de exames mais específicos, porém é indispensável a pesquisa de D-dímero, que é resultado da simultânea coagulação e da fibrinólise, portanto se encontra exacerbado em suspeita de trombose venosa, em contrapartida, baixa taxa de Ddímero dispensa tal suspeita. Foi possível observar uma escassez de literatura específica sobre o desenvolvimento e instalação da trombose. Os estudos sobre a trombose na COVID-19, demonstram que nem sempre essa patologia vai se desenvolver secundária à infecção, porém em pacientes que possuem fatores de risco para o desenvolvimento da trombose as possibilidades de desenvolvimento aumentam.
\end{abstract}

Palavras-chave: COVID-19; Embolia e trombose; Coágulo sanguíneo.

\begin{abstract}
Venous thrombosis is characterized by excessive accumulation of structures composed of fibrins and platelets inside veins, causing partial obstruction or occlusion, usually affecting the lower limbs. The main risk factors for blood ssis, endothelial injury and hypercoagulability states stand out, intensifying with advancing age. Correlate the development of thrombosis in healthy patients and with those affected with COVID-19. The search for scientific articles was carried out in the electronic databases: Scientific Electronic Library Online (SCIELO), Latin American Literature in Health Sciences (LILACS), National Library of Medicine and the National Institute of Health (PubMed). Regarding the occurrence of thrombotic events in COVID-19, thrombosis is believed to be triggered by the "cytokine storm" which is the presence of an exacerbated level of proinflammatory cytokines in the blood. Due to the fact that many conditions present, on clinical examination, a picture similar to those found in thrombosis patients, it is necessary to perform more specific tests, but it is indispensable the research of D-dummer, which is the result of simultaneous coagulation and fibrinolysis, therefore it is exacerbated in suspected venous thrombosis, on the other hand, low ddummer rate dispenses such suspicion. It was possible to observe a scarcity of specific literature on the development and installation of thrombosis. Studies on thrombosis in COVID-19 show that this pathology will not always develop secondary to infection, but in patients who have risk factors for the development of thrombosis the possibilities of development increase.
\end{abstract}

Keywords: COVID-19; Embolism and thrombosis; Blood clot. 


\begin{abstract}
Resumen
La trombosis venosa se caracteriza por la acumulación excesiva de estructuras compuestas por fibrina y plaquetas en el interior de las venas, provocando una obstrucción u oclusión parcial, afectando geralmente a los miembros inferiores. La estasis sanguínea, el daño endotelial y los estados de hipercoagulabilidad se destacan como los principales factores de riesgo, intensificándose con la edad. Correlacionar el desarrollo de trombosis en pacientes sanos y afectados por COVID-19. La búsqueda de artículos científicos se realizó en bases de datos electrónicas: Scientific Electronic Library Online (SCIELO), Literatura Latinoamericana en Ciencias de la Salud (LILACS), Biblioteca Nacional de Medicina e Instituto Nacional de Salud (Pubmed). Con respecto a la aparición de eventos trombóticos en COVID-19, se cree que la trombosis se desencadena por la "tormenta de citocinas", que es la presencia de un nivel exacerbado de citocinas proinflamatorias en la sangre. Decido a que muchas condiciones presentan, en la exploración clínica, un cuadro similar al que se encuentra en los pacientes con trombosis, son necesarias pruebas más específicas, pero la búsqueda del dímero D es fundamental, que es el resultado de la coagulación y la fibrinólisis simultáneas, por lo tanto se agrava en la sospecha de trombosis venosa, por otro lado, una tasa baja de dímero D prescinde de tal sospecha. Fue posible observar una escasez de literatura específica sobre el desarrollo e instalación de trombosis. Los estudios sobre trombosis en COVID-19 muestran que está patología no siempre se desarrollará secundaria a una infección, pero en pacientes que tienen factores de riesgo para el desarrollo de trombosis, las posibilidades de desarrollo aumentan.
\end{abstract}

Palabras clave: COVID-19; Embolia y trombosis; Coágulo sanguíneo.

\title{
1. Introdução
}

A trombose venosa é caracterizada pelo acúmulo excessivo de estruturas compostas por fibrinas e plaquetas no interior de veias, ocasionando obstrução parcial ou oclusão, afetando geralmente os membros inferiores. Porém, por se tratar de doença do sistema circulatório pode ocorrer em qualquer lugar do organismo. Destacam-se como principais fatores de risco a estase sanguínea, lesão endotelial e estados de hipercoagulabilidade, intensificando-se com o avançar da idade (Presti \& Miranda, 2015).

A trombose venosa superficial (TVS) afeta entre de 3 a $11 \%$ da população geral e estudos recentes mostram uma média de prevalência de 18,2\% de trombose venosa profunda (TVP) na população global (Almeida et al., 2019). A estimativa é de 60 casos de TVP para cada 100.000 habitantes por ano (SBACV, 2020). É a terceira doença cardiovascular mais comum no mundo. Nos EUA e na Europa, são relacionadas à TVP cerca de 50 mil mortes por ano, e 300 mil hospitalizações (Flumignan et al., 2018).

A TVP tem início com a formação de um ou mais trombos no sistema venoso profundo, podendo gerar uma série de alterações agudas locais, que determinam suas manifestações clínicas. A longo prazo, uma TVP pode gerar um quadro denominado de Síndrome Pós- Trombótica (SPT), a qual acomete cerca de 20 a 50\% dos pacientes, resultando em uma redução da capacidade produtiva do indivíduo, além de custos elevados para o Sistema de Saúde relacionados ao seu tratamento (Galego et al., 2017).

Em 2020, desencadeou-se a infecção pelo vírus SARS-CoV-2, caracterizada como pandemia de COVID-19 em 11 de março de 2020. No Brasil, o primeiro caso foi confirmado em 26 de fevereiro do mesmo ano no estado de São Paulo (OMS, 2021). De acordo com a Organização Pan-Americana da Saúde (OPAS 2021), foram confirmados 233.503 .524 casos de COVID-19 e 4.777 .503 óbitos em todo o mundo, até o dia 01 de outubro do corrente ano. A região das Américas possui mais de 85 milhões de casos confirmados e o Brasil representa um total de 24\%. Só no Amazonas foram confirmados até 01 de outubro de 2021 com quantitativo de 21.399.546 casos, colocando assim o Estado na $9^{a}$ posição no ranking com a maior mortalidade pela doença e $2^{\mathrm{a}}$ maior taxa de letalidade, com 3,8\% (FVS-AM, 2021)

A infecção pela COVID-19 desencadeia diversas complicações não apenas no sistema respiratório, mas nos demais sistemas do organismo humano, como o circulatório (Guan, 2020). Uma das manifestações clínicas que mais chamou atenção da equipe médica foi a ocorrência de eventos trombóticos, principalmente em pacientes sem fatores de risco conhecidos. Acredita-se que a infecção viral e a resposta inflamatória ao agirem na ativação da cascata de coagulação ocasionou tal evento 
(Viana, et al., 2020). A infecção pela COVID-19 está relacionada ao aumento de citocinas inflamatórias e outras complicações, se comparado aos pacientes hospitalizados em geral (Nascimento et al., 2020).

Sendo assim, este estudo buscará se aprofundar no conhecimento sobre os fatores de risco, bem como o desenvolvimento da trombose. Como observado, em um primeiro momento, a trombose não é doença de uma causa só, portanto é necessário voltar a atenção para os casos que podem ocorrer ou se agravar com o COVID-19, pois o indivíduo infectado pelo novo coronavírus podem apresentar distúrbios de coagulação, assim aumentando o risco de trombose. O trabalho tem por objetivo apresentar através da análise de artigos científicos as principais complicações no desenvolvimento da trombose.

Objetivo geral do trabalho é correlacionar o desenvolvimento da trombose em pacientes sadios e com os que foram acometidos com a COVID-19. Os objetivos específicos delineados são: descrever os fatores determinantes para o desenvolvimento da trombose; observar as causas da trombose em pacientes com a COVID-19 e correlacionar trombose em pacientes com e sem a COVID-19.

\section{Metodologia}

O presente estudo trata-se de uma revisão de literatura com abordagem qualitativa descritiva com o intuito de buscar artigos científicos relacionados ao desenvolvimento da trombose em pacientes com e sem a infecção pelo SARS- CoV-2.

Uma revisão de literatura oferece diferentes formas de realização e é baseada em uma síntese de diferentes partes, com a finalidade de construir um conhecimento científico, pois através de várias teorias surgem oportunidades de novos conhecimentos, com organização e discussão de uma pesquisa baseada em um assunto específico. Consiste basicamente em elaborar uma temática para a pesquisa, buscar na literatura, selecionar os artigos, extrair os dados, avaliar a qualidade metodológica, sintetizar os dados, avaliar a qualidade das evidências e por fim, redigir o texto. As revisões são consideradas secundárias, pois tem sua fonte primária (Carmo \& Souza, 2018).

Foram usados, artigos em inglês, espanhol e português coletados a partir das plataformas digitais Scientific Electronic Library Online (SCIELO), Literatura Latino-Americana em Ciências da Saúde (LILACS), National Library of Medicine and the National Institute of Health (Pubmed), e foram usadas as seguintes palavras chaves em inglês: thrombosis in covid19, thrombosis development, d-dímero and COVID-19, e seus correlatos específicos identificados no Medical Subject Headings (MESH) e em português nos Descritores em Ciências da Saúde (DeCs): COVID-19; Embolia e Trombose; Coágulo Sanguíneo.

Foram incluídas publicações de 2015 a 2021, em idiomas português, espanhol e inglês que estejam disponíveis na íntegra para análise. Foram excluídos estudos com datas anteriores a 2015, fora do contexto e revisões bibliográficas. Foram utilizadas as estratégias de busca conforme descrito no Quadro 1.

Quadro 1 - Estratégia de busca na base de dados.

\begin{tabular}{|l|l|}
\hline \multicolumn{1}{|c|}{ Base de dados } & \multicolumn{1}{c|}{ Estratégias de busca } \\
\hline Scielo & $\begin{array}{l}\text { COVID-19, Trombose e coagulação, profilaxia trombose, eventos da } \\
\text { trombóticos na COVID-19. }\end{array}$ \\
\hline Pubmed & $\begin{array}{l}\text { COVID-19; Desenvolvimento da trombose na COVID-19, trombo } \\
\text { profilaxia, fatores da trombose. }\end{array}$ \\
\hline
\end{tabular}

Fonte: Autores (2021).

Inicialmente, foram encontrados 96 artigos a partir da estratégia de busca pelas bases de dados Scielo, Pubmed E Google Scholar. 70 trabalhos foram selecionados para análise do título e resumo, dos quais 45 artigos foram considerados 
potencialmente relevantes para leitura na íntegra visando garantir maior confiabilidade e validação do material selecionado a ser analisado na revisão de literatura inicial. Foram incluídos no estudo, 28 artigos. O processo de busca e seleção dos estudos está representado na Figura 1.

Figura 1 - Fluxograma de seleção de estudo.

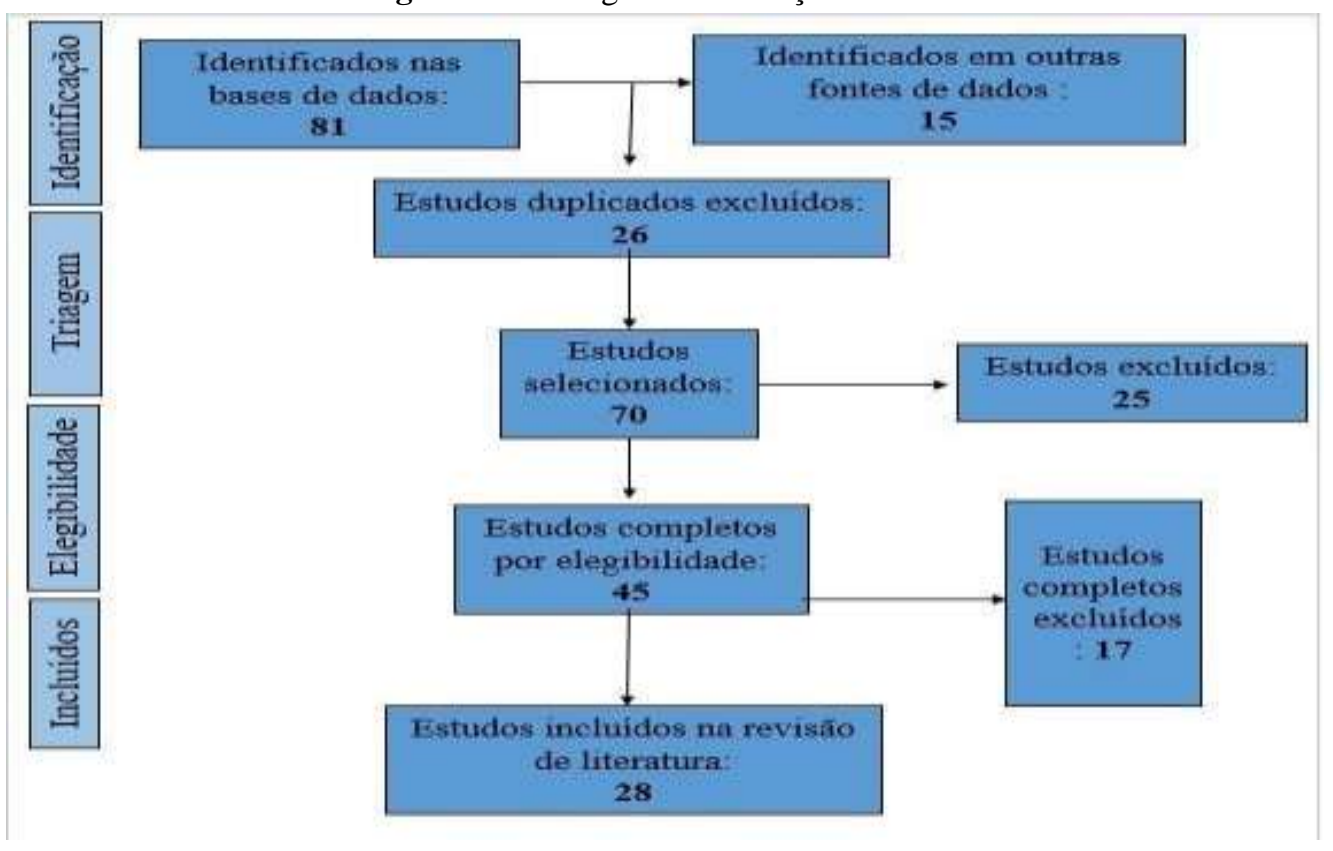

Fonte: Autores (2021).

\section{Resultados e Discussão}

\subsection{Trombose}

A trombose é a formação de um coágulo sanguíneo que ocorre por diversos fatores, ocasionando inflamação na veia e que pode permanecer no local inicial ou se estender gerando obstrução parcial ou total. Quando se desenvolve em veias da superfície corporal, logo abaixo da pele, é chamada de tromboflebite ou flebite, quando em veias profundas, no interior dos músculos, é chamada de trombose venosa profunda ou TVP (Serviço de Linfologia e Feridas, 2021).

A incidência da TVP, segundo alguns estudos, é demonstrada na população geral como 5 casos a cada 10.000 indivíduos anualmente, e no Brasil em torno de 0,6 a cada 1000 habitantes anualmente. Os índices chegam a 600 mil casos de trombose venosa profunda e embolia pulmonar anualmente na Europa. Nos Estados Unidos da América, são registrados 300 mil casos de trombose, já no Brasil, houve 122.096 internações por trombose em 2014, e um decréscimo desse índice para 113.817, em 2015 (Charlo et al., 2020). A prevalência exata de TEV é desconhecida porque se trata de uma patologia de difícil diagnóstico não só pela inespecificidade dos sintomas que apresenta, como também pelo fato de ser assintomática em 50\% dos casos ou a sua única manifestação ser a morte súbita em 25\% de doentes com TEP (Rodrigues et al., 2019).

A fisiopatologia da trombose venosa é descrita pela Tríade de Virchow e consiste em: Danos da parede vascular trauma direto ao endotélio vascular durante procedimentos diagnósticos e terapêuticos; Alterações na velocidade do sangue repouso, imobilização muscular, diminuição de débito cardíaco, compressão venosa, dilatação venosa, são elementos associados à estase venosa; Distúrbio do sangue - congênitos da coagulação, adquiridos pela presença das toxinas de alguns tumores, predisposição à doença tromboembólica venosa por mecanismos hiper coaguláveis e maior viscosidade sanguínea pelo aumento de hematócritos (Farjado et al., 2020).

A TVP é uma doença multifatorial, tendo como fatores de risco mais prevalentes obesidade, neoplasia, cirurgia, imobilização prolongada, tabagismo, TVP prévia. A identificação dos fatores de risco permite contribuir na compreensão sobre 
cada um destes, a fim de melhorar e adequar a eficiência dos diferentes métodos disponíveis para a profilaxia (Natél, 2015). A idade é um importante fator de risco, a incidência aumenta na razão direta da idade (Almeida et al., 2021).

A biologia molecular esclarece as relações entre mediadores da inflamação e o mecanismo da coagulação. O trombo parece aumentar pelo chamamento e localização na sua área das plaquetas e leucócitos ativados, pelos fatores que desencadearam primariamente a trombose vascular, e que induzem depois uma resposta inflamatória. Em condições normais, o sangue apresenta um fluxo laminar, o endotélio normalmente não é reativo aos componentes celulares nem às proteínas da coagulação. Devido a fatores extrínsecos ocorre uma lesão endotelial, em resposta a esta lesão o equilíbrio trombohemorrágico altera-se favorecendo a produção de trombina e a formação do trombo, segue-se a ampliação do trombo mediada por selectinas (Alves et al., 2015).

\subsection{Sars-COV-2}

O primeiro relato sobre o novo coronavírus ocorreu em Wuhan, na província de Hubei, na China, em 12 de dezembro de 2019, 45 dias após a internação do primeiro paciente já eram registrados 1975 casos, o que já demonstrava o seu alto potencial de contaminação ( $\mathrm{Wu}, 2020)$. Foi considerada inicialmente uma pneumonia causada por vírus que tem como principais sintomas febre, tosse seca, cansaço e dor de cabeça, por ser muito agressivo causa danos alveolares ocasionando insuficiência respiratória (Zhou, 2020). Pode também ser assintomático, apresentar quadros mais graves como sintomas gastrointestinais e insuficiência renal, ou ainda progredir para uma pneumonia ou óbito. Seu índice de fatalidade geralmente está associado à idade avançada ou a presença de comorbidades, o que por si só já demonstra vulnerabilidade do hospedeiro (Carvalho et al., 2020).

O vírus tem grande afinidade com a enzima conversora de angiotensina 2 (ECA2), abundante nas células epiteliais pulmonares e é através dela que adentra as células humanas. A proteína $\mathrm{S}$ presente no vírus é reconhecida pela ECA2 que permite sua entrada na célula, iniciando assim a infecção, a partir daí o vírus começa a replicar poli proteínas utilizando os ribossomos da célula (Silva et al., 2021).

Vários estudos demonstram que o vírus pode usar toda a proteína Eca-2 presente nas células atacadas, exacerbando assim a ação da angiotensina II que irá lesionar tecidos e órgãos, como a Eca 2 é presente em várias células de vários sistemas, isso leva ao risco de outros órgãos além dos pulmões serem atacados, então dessa maneira, o vírus pode gerar múltiplas lesões por todo o organismo humano (Batschauer \& Jovita, 2020). Podemos ter uma visão geral e resumida sobre o COVID-19 na Figura 2. 
Figura 2 - A origem, transmissão, apresentação e complicações clínicas da Pandemia SARS-Cov-2 (COVID-19).

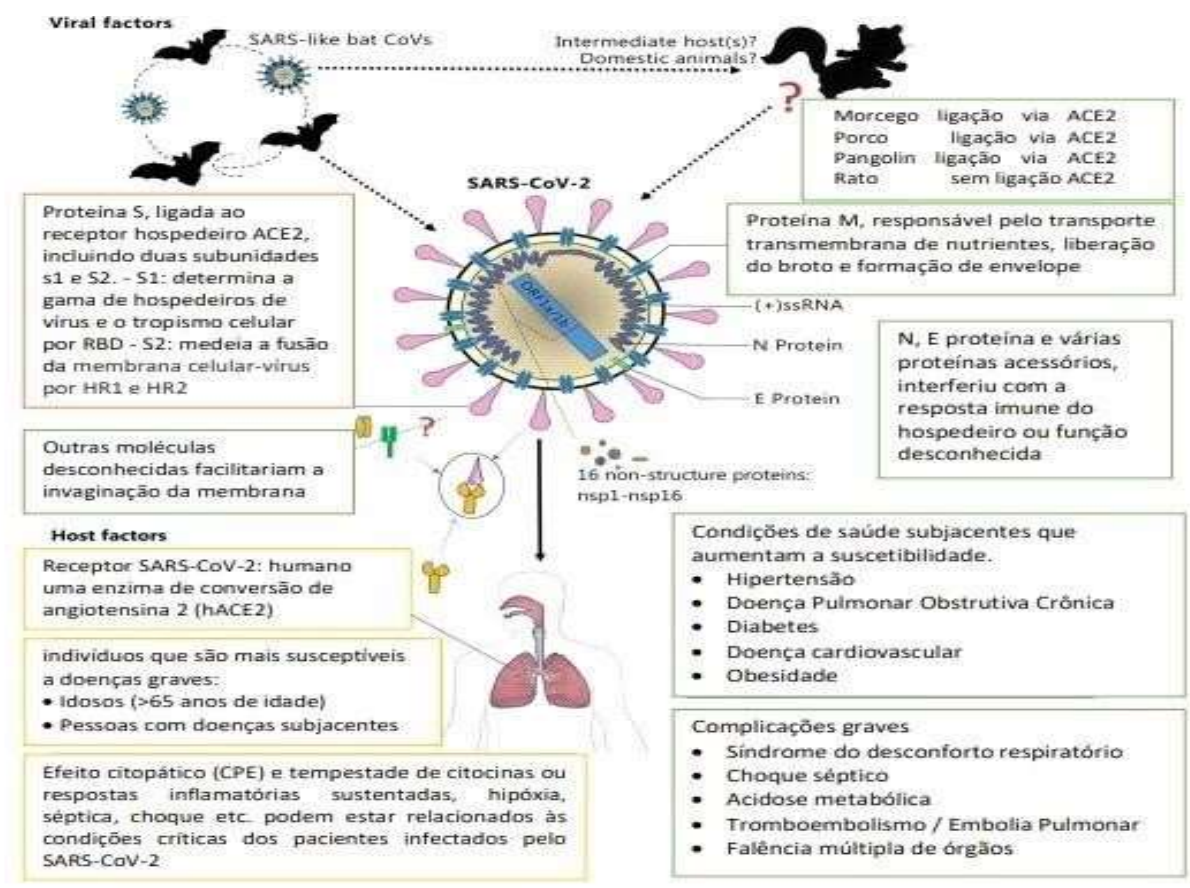

Fonte: Guo, et al (2020).

De acordo com a OPAS (2020), uma das complicações clínicas que se manifestam em pacientes infectados pelo COVID-19 são os eventos trombóticos, a cada 9 casos, 5 apresentam esta complicação. Acredita-se que isso se deva aos danos causados à hemostasia e dos mecanismos imunológicos desencadeados durante o processo de patologia da COVID-19 (Cantamissa et al., 2020).

\subsection{Correlação COVID-19 X trombose}

Em se tratando da ocorrência de eventos trombóticos na COVID-19, acredita-se que a trombose seja desencadeada pela "tempestade de citocinas" que é a presença de um nível exacerbado de citocinas pró-inflamatória no sangue. A infecção ocasiona uma intensa resposta inflamatória, devido ao fato de a inflamação induzir a ativação da coagulação e em contrapartida, a coagulação potencializa a atividade inflamatória, conforme podemos ver na Figura 3 (Nascimento et al, 2020): 
Figura 3 - Ativação da coagulação.

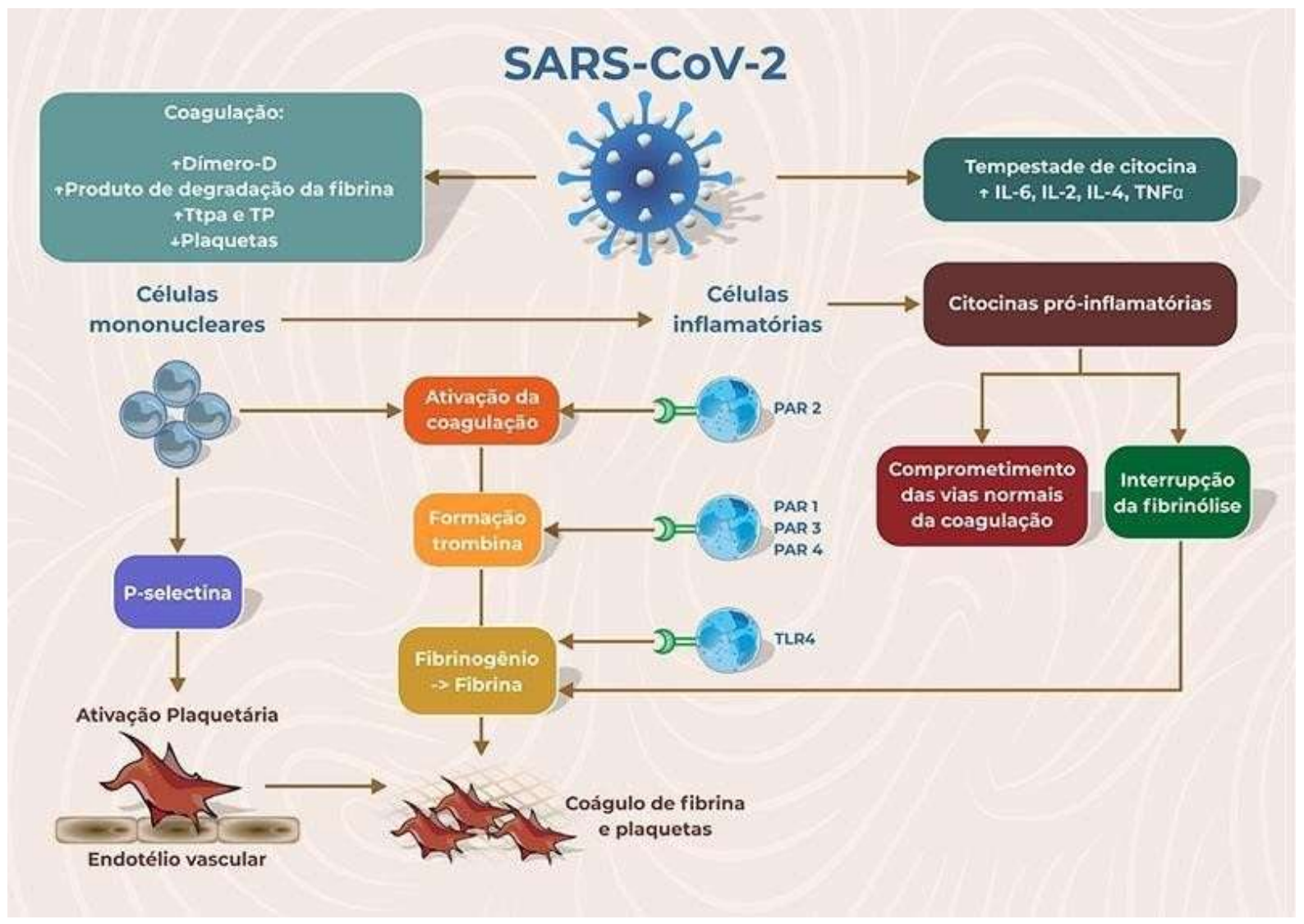

Fonte: Adaptada LEVI (2020).

A liberação de alta quantidade de mediadores inflamatórios na corrente sanguínea e terapêutica com hormônios e imunoglobulinas aumentam a viscosidade do sangue, em combinação com ventilação mecânica, cateterismo venoso central ou cirurgia, que podem causar lesão endotelial vascular, ocasionam o surgimento de trombose venosa profunda (Fleury, 2020).

O Quadro 2, mostra os resultados dos estudos analisados para a correlação da trombose e a COVID-19: 
Quadro 2 - Principais resultados encontrados.

\begin{tabular}{|c|c|c|}
\hline Autor/Ano & Título & Resultados \\
\hline Almeida et al., 2021 & $\begin{array}{l}\text { Fatores desencadeantes de } \\
\text { tromboembolismo } \\
\text { venoso. }\end{array}$ & $\begin{array}{l}\text { A estase venosa, a injúria da parede celular e a coagubilidade sanguínea aumentada favorecem o TEV, } \\
\text { surgindo então, a tríade de Virchow: lesão endotelial, lentificação do fluxo sanguíneo e aumento na } \\
\text { coagubilidade do sangue. Os dois primeiros componentes relacionam-se a fatores de risco adquiridos, } \\
\text { enquanto a hipercoagubilidade sanguínea possui principalmente causas genéticas. }\end{array}$ \\
\hline Barbosa et al., 2021. & $\begin{array}{l}\text { Trombose terial em } \\
\text { microcirculação pós- } \\
\text { Covid Relato de caso 19: }\end{array}$ & $\begin{array}{l}\text { O estado de hipercoagubilidade e coagulação intravascular disseminada, provocada pela entrada do vírus } \\
\text { nas células estão relacionados à associação do SARS-CoV-2 com um alto potencial para causar } \\
\text { trombose da microcirculação arterial em pacientes. }\end{array}$ \\
\hline $\begin{array}{l}\text { Batschauer \& Jovita, } \\
2020 .\end{array}$ & $\begin{array}{l}\text { Hemostasia e COVID-19: } \\
\text { fisiopatologia, exames } \\
\text { laboratoriais e terapia } \\
\text { anticoagulante. }\end{array}$ & $\begin{array}{l}\text { A coagulopatia na COVID-19 é um evento frequentemente descrito e está associada a um estado de } \\
\text { hipercoagulabilidade. A lesão celular causada pela entrada do vírus SARS-CoV-2, aumenta a resposta } \\
\text { inflamatória, com liberação de mais citocinas e fator de necrose tumoral e consequente estímulo pró- } \\
\text { coagulante. A resposta imunológica inata aos processos infecciosos também pode estar ligada ao estado } \\
\text { pró coagulante da COVID-19 através da ação, por exemplo, do fator tecidual. }\end{array}$ \\
\hline $\begin{array}{l}\text { Cantamissa et al., } \\
2020\end{array}$ & \begin{tabular}{|l|} 
Relato de caso: trombose \\
venosa periférica como \\
consequência secundária a \\
infecção do novo \\
coronavírus. \\
\end{tabular} & $\begin{array}{l}\text { Na permanência do novo coronavírus no organismo, há considerável e oscilante desordens vasculares } \\
\text { desencadeadas pela tempestade de citocinas pró inflamatórias e pela perturbação dos anticoagulantes no } \\
\text { período de manifestação da patologia. Durante a infecção, a resposta inflamatória exacerbada sistêmica, } \\
\text { simultaneamente, a hipóxia provoca perturbação endotelial e aumento da atividade pró trombótica. }\end{array}$ \\
\hline Charlo et al., 2020. & $\begin{array}{l}\text { Relação entre trombose } \\
\text { venosa profunda e seus } \\
\text { fatores de risco na } \\
\text { população feminina. }\end{array}$ & $\begin{array}{l}\text { O aumento da estase sanguínea, por um período longo de imobilização, lesões do vaso sanguíneos de } \\
\text { maneira direta ou lesão endotelial pela passagem de cateter venoso central, ativam a cascata da } \\
\text { coagulação, evoluindo para quadro patológico. Também foi observado relação nociva entre trombose e } \\
\text { cigarro, gera dano na parede vascular, com redução do oxigênio local, elevação dos níveis de } \\
\text { fibrinogênio plasmático, ativação da cascata de coagulação, e a partir da nicotina, indução de um estado } \\
\text { pró trombótico por ativação plaquetária, levando a TVP. }\end{array}$ \\
\hline Gomes, 2020. & $\begin{array}{l}\text { Embolia Pulmonar } \\
\text { Venosa Profunda: uma } \\
\text { revisão bibliográfica }\end{array}$ & $\begin{array}{l}\text { Os principais fatores de risco para o desenvolvimento da trombose venosa estão relacionados à } \\
\text { imobilidade uso de anticoncepcionais com estrógeno aumenta até quatro vezes o risco da doença, a idade } \\
\text { superior a } 40 \text { anos corresponde a } 89 \% \text { dos casos, infarto do miocárdio e as varizes por sua vez } \\
\text { comprometem a circulação sanguínea favorecendo a formação de Coágulos. É física como no caso de } \\
\text { paralisia, pacientes acamados, gestação e pós-parto. }\end{array}$ \\
\hline Melo et al., 2021. & $\begin{array}{l}\text { Aspectos epidemiológicos } \\
\text { sobre COVID-19 e a } \\
\text { relação com trombose } \\
\text { venosa em tempos de } \\
\text { pandemia: uma revisão de } \\
\text { literatura. }\end{array}$ & $\begin{array}{l}\text { Idade associada a cirurgias e longos períodos de imobilização são fatores de risco para o } \\
\text { desenvolvimento da trombose. Neste sentido, os enfermos com COVID-19 em tratamento respiratório } \\
\text { intensivo e uso de ventilação mecânica ou extracorpórea, possuem mais chances de apresentar TV na } \\
\text { forma embolia pulmonar. }\end{array}$ \\
\hline Moreira et al., 2021. & $\begin{array}{l}\text { Tromboembolismo } \\
\text { pulmonar: dos aspectos } \\
\text { epidemiológicos ao } \\
\text { tratamento. }\end{array}$ & $\begin{array}{l}\text { Os fatores de risco são determinados pelos componentes da Tríade de Virchow: lesão endotelial, } \\
\text { alterações do fluxo sanguíneo normal e um estado de hipercoagulabilidade. Dessa forma, situações de } \\
\text { imobilização prolongada, como cirurgias ortopédicas, viagens aéreas de longas distâncias, são } \\
\text { predisponentes para o desenvolvimento do TEP. Reincidência de quadros de TEP predispõe outras } \\
\text { manifestações, por causa das alterações endoteliais provocadas pelo episódio inicial. Uso de } \\
\text { anticoncepcional, gravidez e puerpério são situações de risco para o TEP causadas por estase venosa e } \\
\text { por alterações hematológicas, como o aumento do nível de fatores de coagulação e do fibrinopeptídeo A. }\end{array}$ \\
\hline Silva et al., 2021. & $\begin{array}{l}\text { COVID-19: Aspectos da } \\
\text { origem, fisiopatologia, } \\
\text { imunologia e tratamento - } \\
\text { uma revisão narrativa. }\end{array}$ & $\begin{array}{l}\text { A infecção estimula a resposta imunológica complexa nos pacientes, onde os agentes pró e } \\
\text { antinflamatórios irão contribuir para eliminar o processo infeccioso e recuperar o tecido. Portanto, há } \\
\text { evidências concretas da associação da virulência da COVID-19 com fenômenos de coagulação } \\
\text { intravascular que poderão evoluir par TVP. }\end{array}$ \\
\hline
\end{tabular}

Fonte: Autores (2021).

Tanto a trombose em pacientes não infectados, quanto em pacientes com COVID-19 se desenvolve a partir do surgimento da Tríade de Virchow. Em pacientes comuns, cada componente da Tríade de Virchow pode ser adquirido isoladamente, como lentidão do fluxo sanguíneo através de cirurgias, imobilidade por hospitalização; lesão endotelial por uso de cateter, ou histórico de TEV; e coagulopatia por causas genéticas (Almeida et al., 2021; Charlo et al., 2020; Gomes, 2020; Moreira et al., 2021).

Na COVID-19 os componentes da Tríade de Virchow podem ser desencadeados apenas pela infecção pelo SAR-CoV2, a lesão endotelial é causada pela entrada do vírus na célula, a hipóxia também provoca perturbação endotelial; o fluxo sanguíneo se torna lento devido ao longo período de internação para o tratamento da infecção; a tempestade de citocinas" irá ativar os agentes pró e antinflamatórios gerando o estado de hipercoagubilidade e coagulação intravascular que poderão evoluir para TVP (Barbosa et al., 2021; Batschauer \& Jovita, 2020; Cantamissa et al., 2020; Melo et al., 2021; Silva et al., 2021).

Pelo fato de muitas afecções apresentarem, ao exame clínico, quadro semelhante aos encontrados em pacientes de trombose, é necessária a realização de exames mais específicos, como ultrassonografia, exame de sangue, venografia, eco color doppler, tomografia e ressonância magnética, ainda assim, tais exames tem baixa sensibilidade e nem sempre é possível 
identificar a ocorrência do evento. Porém é indispensável a pesquisa de D-dímero, que é resultado da simultânea coagulação e da fibrinólise, portanto se encontra exacerbado em suspeita de trombose venosa, em contrapartida, baixa taxa de D-dímero dispensa tal suspeita (Cercas, 2017).

Estudos demonstraram que os níveis de coagulação sanguínea em pacientes com COVID-19 se encontram alterados, mais especificamente os valores do dímero D aumentam significativamente enquanto de outros marcadores ficam baixos, o que torna essencial seu monitoramento de rotina, pois se apresenta como um marcador confiável da evolução do quadro (Silveira, 2020).

Ainda de acordo com Cercas (2017), o tratamento da trombose se fundamenta na anticoagulação e na fibrinólise com o objetivo de evitar recorrências e evolução para tromboembolismo pulmonar, preservando as válvulas cardíacas. Porém devese levar em conta o tipo, local e outras afecções existentes, a fim de se escolher o melhor medicamento, evitando assim, a ocorrência de sangramentos e outras complicações. Pois a literatura não indica nenhum anticoagulante $100 \%$ seguro em relação a hemorragias, cada um apresenta riscos diferente que estão relacionados a questões como duração do tratamento, posologia, associação com outros medicamentos, condições do paciente, entre outros fatores (SBACV, 2015).

\section{Considerações Finais}

Mesmo a trombose sendo uma questão de saúde pública, pois é uma das doenças cardiovasculares que mais levam ao óbito no mundo, é possível observar uma escassez de literatura específica sobre essa doença. Seja por seu difícil diagnóstico, ou por ser multifatorial e assintomática na maioria dos casos. Foi possível observar que após seu desenvolvimento, vai gerando inúmeras complicações, visto que o trombo pode se locomover dentro dos vasos sanguíneos e se instalar em qualquer parte do organismo humano.

Os estudos encontrados sobre a trombose na COVID-19, demonstram que nem sempre essa patologia vai se desenvolver secundária à infecção, porém, pacientes que possuem fatores de risco para o desenvolvimento da trombose precisam de uma atenção redobrada e medidas de profilaxia adequadas, visto que características fisiopatológicas da COVID-19 exacerbam as possibilidades de seu desenvolvimento. E como já dito, esta pode acarretar risco maiores quando se instalam em pacientes infectados, pois podem chegar aos pulmões que são muito afetados pelo vírus.

Então, é importante que haja mais incentivo nas pesquisas sobre todo o desenvolvimento e instalação da trombose no organismo humano, para que assim possam ser criadas políticas públicas efetivas de combate e tratamento desta patologia que tem grande impacto na vida dos pacientes bem como na saúde pública do país.

Assim novos estudos a serem feitos de caráter prospectivos longitudinais, que visem acompanhar esses pacientes quanto a evolução para eventos trombóticos, assim como a busca de marcadores plasmáticos preditores de efeitos trombóticos são de suma importância para garantir tratamento precoce e melhora no prognóstico.

\section{Referências}

Almeida, M. J. D., Guillaumon, A. T., Miquelin, D., Joviliano, E. E., Hafner, L., Sobreira, M. L., \& Yoshida, W. B. (2019). Diretrizes de conceito, diagnóstico e tratamento da trombose venosa superficial. Jornal Vascular Brasileiro, 18.

Almeida, N. R., Pereira, L. D. L., \& de Oliveira Alvim, H. G. (2021). Fatores desencadeantes de tromboembolismo venoso. Revista JRG de Estudos Acadêmicos, 4(8), 213-221.

Barbosa, J. V. C., Rodrigues, P. F., de Lima, C. V. B. Q., Neto, O. J. F., Mendes, I. P. G., Pereira, A. C., \& Neto, E. D. C. M. (2021). Trombose arterial em microcirculação pós-Covid 19: Relato de caso. Research, Society and Development, 10(4), e50410413857-e50410413857...

Batschauer, A. P., \& Jovita, H. W. (2020). Hemostasia e COVID-19: fisiopatologia, exames laboratoriais e terapia anticoagulante. A Tempestade do Coronavírus, 52(2), 138-42.

Cantamissa, M. L. D., Almeida, W. C. P., Silva, E. F. G., Oliveira, J. M. F., Silva, L. C. F., \& da Silva, J. S. (2021). Relato De Caso: trombose venosa periférica como consequência secundária a infecção do novo coronavírus. Anais do Seminário Científico do UNIFACIG, (6). 
Carvalho, F. R., Gobbi, L. C., Carrijo-Carvalho, L. C., Caetano, A. J. F., Casotti, G. C., Tiussi, L. M., \& Cavalari, A. L. C. (2020). Fisiopatologia da COVID-19: repercussões sistêmicas. Unesc em Revista, 4(2), 170-184.

Cercas, M. D. R. B. (2017). Trombose venosa: revisão a propósito de caso clínico. Dissertação (Mestrado Integrado Medicina) - Universidade de Lisboa. Faculdade de Medicina de Lisboa.

Charlo, P. B., Herget, A. R., \& Moraes, A. O. (2020). Relação entre trombose venosa profunda e seus fatores de risco na população feminina. Global Academic Nursing Journal, 1(1), e10-e10.

Charlo, P. B., Herget, A. R., \& Moraes, A. O. (2020). Relação entre trombose venosa profunda e seus fatores de risco na população feminina. Global Academic Nursing Journal, 1(1), e10-e10.

Fleury, M. K. (2020). A COVID-19 e o laboratório de hematologia: uma revisão da literatura recente. Revista Brasileira de Análises Clínicas, 52(2), 131-7.

Flumignan, R.L.G.; Amaral, F.C.F, \& Flumignan, C.D.Q (2018). Trombose Venosa Profunda. 2018. Proterapeutica, 7.

Fundação de Vigilância Sanitária do Amazonas (FVS-AM) (2021). Situação Epidemiológica da COVID-19 no estado do Amazonas. $<$ https://www.fvs.am.gov.br>.

Galego, G., Silveira, P. G., Franklin, R. N., Bortoluzzi, C. T., da Silva Eli, E., \& Broering, J. J. (2017). Tratamento da trombose venosa profunda aguda com rivaroxabana. Arquivos Catarinenses de Medicina, 46(1), 68-79.

Gomes, E. (2021). Embolia pulmonar venosa profunda: uma revisão bibliográfica. Trabalhos de Conclusão de Curso. (Bacharel de Enfermagem) - Centro Universitário UNIFACIG, Manhuaçu, 2020.

Guan, W. J., Ni, Z. Y., Hu, Y., Liang, W. H., Ou, C. Q., He, J. X., \& Zhong, N. S. (2020). Clinical characteristics of coronavirus disease 2019 in China. New England journal of medicine, 382(18), 1708-1720.

Moreira, M. V., de Oliveira Vieira, J. D., dos Santos, A. B. B., Netto, A. F., de Mendonça Fonseca, I. G. N., Lopes, J. A., \& Tavares, R. L. (2021). Tromboembolismo pulmonar: dos aspectos epidemiológicos ao tratamento. Brazilian Journal os Health Review, 4(2), 8350-8363.

Nascimento, J. H. P., Gomes, B. F. D. O., Carmo, P. R. D., Petriz, J. L. F., Rizk, S. I., Costa, I. B. S. D. S., \& Oliveira, G. M. M. D. (2020). COVID-19 e estado de hipercoagulabilidade: uma nova perspectiva terapêutica. Arquivos Brasileiros de Cardiologia, 114, 829-833.

Natél, E. C. (2015). Análise dos fatores de risco de trombose venosa profunda em pacientes atendidos pela cirurgia vascular, no Hospital do Servidor Público Municipal em São Paulo. Trabalho de Conclusão de Curso (Bacharel em Residência Médica) - Comissão de Residência Médica do Hospital do Servidor Público Municipal, 2020.

Organização das Mundial de Saúde (2021). Coronavirus disease (COVID-19) pandemic. https://who.int/emergencies/diseses/novel-coronavirus-2019.

Organização Pan-Americana da Saúde (2020). Alerta Epidemiológico. Complicações e sequelas da COVID-19. https://www.paho.org.

Organização Pan-Americana da Saúde (2021). Situação Epidemiológica. https://covid19.who.int.

Presti, C. \& Miranda Jr, F (2015). Projeto Diretrizes Sociedade Brasileira de Angiologia e Cirurgia Vascular. Trombose Venosa Profunda. Diagnóstico e Tratamento.

Rodrigues, L. G., Matiles, R. S., Rodrigues, M. R., Melo, L. M. F., dos Santos Alvarenga, K. A., \& Leite, S. A. (2019). Perfil epidemiológico de embolia e trombose arteriais no município de Manhuaçu/Mg. Anais do Seminário Científico do UNIFACIG, (5).

Santos, M. H. B., \& Moita, A. N. C. (2021). Aspectos epidemiológicos sobre COVID-19 e a relação com trombose venosa em tempos de pandemia: uma revisão de literatura. -Revista Científica Multidisciplinar, 2(6), e26508-e26508.

Serviço De Linfologia E Feridas. Trombose Venosa Profunda (2021). www.trombose.med.br.

Silva, C. C., de Carvalho, C. M. O., de Lima, D. C., Costa, E. S., de Andrade, V. M. B., Tenorio, B. M., \& Tenorio, F. C. A. M. (2021). COVID-19: Aspectos da origem, fisiopatologia, imunologia e tratamento-uma revisão narrativa. Revista Eletrônica Acervo Saúde, 13(3), e6542-e6542.

Silveira, A. C. (2020). Impacto do micro bioma na COVID-19. Revista Brasileira de Análises Clínicas, 52(2), 192-3.

Sociedade Brasileira de Angiologia e de Cirurgia Vascular (2021). Estimativa SBACV. https://sbacv.org.br/imprensa/estimativas/.

Sociedade Brasileira de Angiologia e de Cirurgia Vascular. Projeto Diretrizes. Trombose Venosa Profunda: Diagnóstico e Tratamento (2021). https://sbacv.org.br/profissionais-da-saude-/diretrizes/.

Viana, T., Bezerra, M. L. B. G., Melo, R. M. V. D., Bezerra, C. G., Mamédio, V., Dourado, G. P., \& Passos, L. C. S. (2021). Infarto Agudo do Miocárdio com Trombose Coronária em um Paciente com COVID-19 sem Fatores de Risco para Doença Cardiovascular. Arquivos Brasileiros de Cardiologia, 116, $511-515$.

Wu, F., Zhao, S., Yu, B., Chen, Y. M., Wang, W., Song, Z. G., \& Zhang, Y. Z. (2020). A new coronavirus associated with human respiratory disease in China. Nature, 579(7798), 265-269.

Zhou, P., Yang, X. L., Wang, X. G., Hu, B., Zhang, L., Zhang, W., \& Shi, Z. L. (2020). A pneumonia outbreak associated with a new coronavirus of probable bat origin. Nature, 579(7798), 270-273. 